\title{
Luz solar en recintos urbanos de alta densidad edilicia en el modelo oasis de la ciudad de Mendoza. Aspectos cuantitativos y cualitativos [período estival]
}

Córica, María Lorena

Arquitecta, egresada de la Facultad de Arquitectura y Urbanismo (FAU), Universidad de Mendoza. Doctora en Medio Ambiente Visual e Iluminación Eficiente, Facultad de Ciencias Exactas y Tecnología, Universidad Nacional de Tucumán. Magíster en Arquitectura de Zonas Áridas y Sísmicas, Facultad de Arquitectura y Diseño. Universidad Nacional de San Juan. Investigadora adjunta CONICET. Jefa de Trabajos Prácticos de la cátedra Diseño Urbano Sustentable II, carrera de Arquitectura, Facultad de Ingeniería, Universidad Nacional de Cuyo. Pertenencia institucional: INAHE (Instituto de Ambiente, Hábitat y Energía) - CONICETCCT Mendoza.lcorica@mendoza-conicet.gob.ar

Ruiz, María Angélica

Ingeniera agrónoma, egresada de la Facultad de Ciencias Agrarias, UNCuyo. Doctora en Ciencias, Área Energías Renovables, Facultad de Ciencias Exactas, UNSa. Tema de tesis: Efectos microclimáticos de la vegetación en ciudades de zonas áridas. Incidencia sobre los consumos energéticos y la calidad ambiental del hábitat. Investigadora asistente CONICET: Tipologías verdes en entornos urbanos de zonas áridas. Determinación de indicadores de eficiencia ambiental. Arbolado en alineación. Pertenencia institucional: INAHE (Instituto de Ambiente, Hábitat y Energía) - CONICET- CCT Mendoza. aruiz@mendoza-conicet.gob.ar

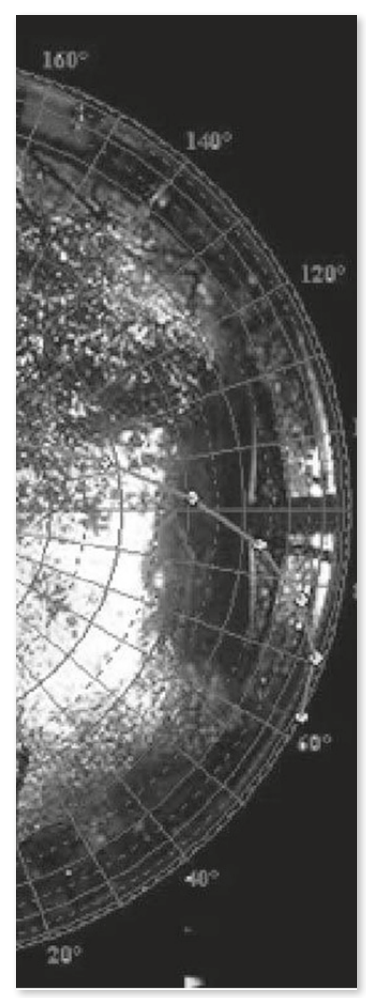

http://dx.doi.org/10.30972/crn.25253516 ISNN 1666-6186. Volumen 25 N. ${ }^{\circ} 25$ (Noviembre de 2018) Pp. 117-138 - Recibido: el 08-06-18. Aprobado: 05-11-18 


\title{
Resumen
}

La ciudad de Mendoza, que se ubica en una región semiárida, se compone por una red de canales viales articulada con arbolado en alineación, sistema conocido como modelo de ciudad oasis. En este ambiente, la incidencia de la iluminación natural se ve condicionada por las características de todos los elementos que participan en los recintos urbanos. El objetivo del presente trabajo es realizar un estudio cuali-cuantitativo de las condiciones lumínicas durante el verano. Se evalúa la luz natural en dos casos de estudio característicos de la ciudad, mediante técnicas combinadas de mediciones in situ y valoraciones subjetivas de los usuarios. Los resultados obtenidos de confort visual en espacios exteriores han determinado que los sujetos se encuentran adaptados al clima luminoso de la región. En particular, se han adaptado a las diferentes condicionantes que genera la morfología edilicia y, consecuentemente, a la diversidad de contrastes que puede arrojar el espacio urbano.

\section{Palabras clave}

Comportamiento de la luz natural; calles arboladas; morfología urbana; confort visual.

\begin{abstract}
Solar insolation in high density urban areas in the oasis model of the city of Mendoza. Quantitative and qualitative dimensions during the summer season.

Mendoza's City, which is located in a semi-arid region, is composed of a network of urban canyons articulated with aligned trees, a system known in this environment as the oasis city model, where the incidence of the natural light is conditioned by the all the design elements that characterize these urban enclosures. The objective of this study is to perform the qualitative and quantitative study of daylight conditions during summer. The incidence of natural light is evaluated in two typical study cases of the city, using combined techniques of in situ measurements and subjective assessments of neighbors. The results obtained of visual comfort in outdoor spaces determine that the design elements are adapted to the luminous climate of the region. In particular, they are adapted to the different constraints generated by building morphology and, consequently, to the diversity of contrasts that urban space can allow.
\end{abstract}

\section{Keywords}

Sunlight performance, tree-lined streets, urban morphology, visual comfort. 


\section{Introducción}

La performance de la luz solar está determinada por las características de dos variables relacionadas con la luz natural: las particularidades del clima regional y la morfología del ambiente construido. La primera considera la disponibilidad real de la luz natural en el ambiente natural. La segunda toma al recinto urbano como determinante del desempeño luminoso del espacio construido. En cuanto al aspecto de morfología urbana, la disponibilidad final de luz natural que tendrán los espacios está influenciada por las características arquitectónicas del recinto urbano (Mascaró, 1983). Por lo tanto, el ambiente natural y el construido son fuerzas birregionales que influyen en el potencial de iluminación natural en los espacios (Guzowski, 2001). La magnitud relativa de las componentes difusa (visión de cielo) y reflejada (edificación circundante) de la radiación está fuertemente condicionada por las particularidades de los recintos urbanos conexos, en aspectos de densidad, altura de las edificaciones, dimensiones, arbolado y reflectividad de las superficies, entre otros (TREgenZA, 1995: 83- 89; TSAngrassoulis Et ÁL., 1999: 439-446; Fontoynont, TSANGrassoulis y SynNEFA, 2004).

En regiones áridas, los elevados niveles de radiación solar diarios y anuales determinan condicionantes ambientales rigurosas y hostiles. Sin embargo, y precisamente por esta característica, la respuesta de acondicionamiento del hábitat se ha centrado en la búsqueda de las sombras como elemento de control ambiental, para evitar los deslumbramientos desde la perspectiva del confort visual, y la mitigación del calor estival desde la perspectiva del confort térmico. Sin una mediación morfológica, estos ambientes serían inhabitables ${ }^{1}$.

La ciudad de Mendoza ( $32^{\circ} 53^{\prime} \mathrm{S}, 68^{\circ}$ 51’ $\mathrm{W}, 750 \mathrm{msnm}$ ), situada en la región centro-oeste de la República Argentina, presenta un clima semiárido, por lo que el $83 \%$ del año corresponde a cielo despejado o parcialmente despejado. El paisaje urbano determina un "modelo de ciudad oasis"2, donde el árbol y sus períodos de foliación se convierten en el elemento de mitigación estacional. Como impronta urbana, el arbolado da una respuesta particular a las formas de ocupación del territorio, modificando el paisaje natural con la disposición de un
1. Pearlmutter en su trabajo "Microclimatic analysis of compact urban canyons in an arid zone" determina que no es sorprendente encontrar asentamientos urbanos en regiones áridas, construidos a espaldas de su entorno, que se encierran negando la dureza del desierto, y que intentan implantar alguna forma urbana que sirva como un oasis en este medio. El fenómeno se fundamenta en la necesidad del hombre de optimizar y mejorar las condiciones de confort proyectando morfologías adecuadas a los contrastes climáticos que impactan en las actividades de la vida, como vivir, trabajar y descansar.

2. El modelo urbano de la ciudad oasis de Mendoza es producto de diferentes procesos históricos que posibilitaron la superposición de distintas redes: una matriz hídrica de sistemas de riego (canales y acequias) desarrollados por los aborígenes prehispánicos, sobre la cual en la etapa fundacional se plasma la traza damero y a partir de la interacción de ambas surge una nueva malla relacionada con la aparición de los forestales (PONTE, 1987: 42-43.). Las acequias fueron elementos característicos de la ciudad desde sus comienzos, delineando ciertos criterios de planificación. Pocas ciudades en el mundo han desarrollado un sistema de riego que se entremezcla a lo largo de la ciudad con la forestación. Mendoza, sostiene un verdadero bosque urbano que protege totalmente a la ciudad de la aridez colindante. Este aspecto particular en torno al arbolado conlleva a la suposición de un amplio conocimiento en la utilización del árbol como elemento estético y fundamentalmente ambiental. 
verdadero bosque urbano como estrategia de confort bioclimático. En este modelo y para la estación calurosa, el efecto principal es la sombra que conforma túneles naturales de distintas permeabilidades lumínicas consolidados por el bosque-urbano. El trazo de verde genera espacios umbríos que evidencian una ciudad "oscura". La arboleda urbana define escenarios lumínicos, espacios oscuros y bajos niveles de luz natural, ante las sombras que proyectan la arboleda y la arquitectura construida, donde el aporte de la componente reflejada pierde importancia como fuente potencial en la iluminación natural. En la búsqueda de situaciones de confort provistas por la sombra en el entorno urbano, el bloqueo de la radiación solar directa disminuye y modifica considerablemente la luz natural disponible como potencial fuente de energía luminosa en los espacios conexos (CóricA, 2010).
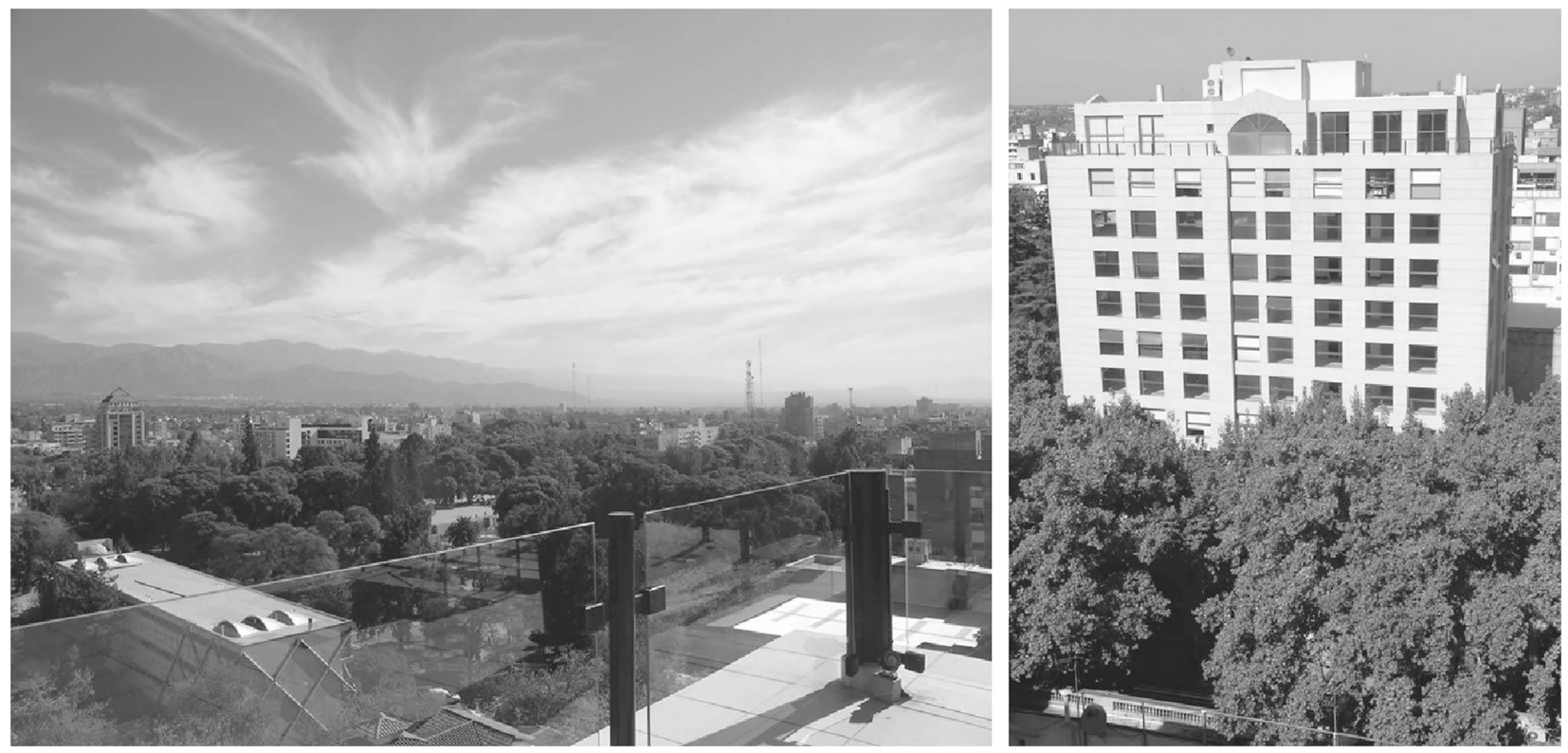

Figura 1. Vista de la ciudad oasis de Mendoza. Fuente: elaboración propia 
Una de las principales características de la luz natural es su variabilidad, tanto en magnitud como en contenido espectral y distribución, la ocurrencia según los momentos del día y del año y de las trayectorias solares (Boyce y STANFord, 2000: 617-650). El acto de ingresar o salir de un edificio, caminar por una senda peatonal soleada o en sombra son ejemplos cotidianos para poner de manifiesto la cantidad de circunstancias cambiantes en el sistema visual, que van a influir a la hora de realizar el desplazamiento o la permanencia y la posibilidad de disfrutar de los espacios exteriores (CANTALEjo Cano, 2001). Existen otros factores influyentes, como es el caso del confort visual, y que pueden ser tanto de orden cualitativo como cuantitativo (Compagnon, 2004: 321-328).

En la actualidad, es un hecho incuestionable la necesidad de evaluar funcionalmente al individuo para así obtener datos de su rendimiento visual ante la ejecución de determinadas tareas (Apple, Apple y Blasch, 1980: 187-224). Cuando un sujeto se desplaza, se ve afectado por diferentes variables de tipo medioambiental (condiciones de contraste, tamaño de los objetos, “desorden visual” del entorno, superficies deslumbrantes, brillos, reflejos etc.). Quizás una de las más críticas a la hora de pronosticar su rendimiento y seguridad durante el período diario y en el espacio exterior sea la derivada de los efectos de las condiciones de iluminación presentes en cada momento y para cada región, según la capacidad de adaptación al clima luminoso (GoyetTe ET ÁL., 2004).

Ante lo expuesto y frente a esta complejidad urbano-arquitectónica que se da en la ciudad, el presente trabajo propone realizar un estudio integral de las condiciones lumínicas en escenarios de diferentes configuraciones dentro de la micro-escala urbana. Por una parte, analizar el comportamiento de luz diurna de espacios exteriores en tejidos urbanos típicos de la alta densidad edilicia y relacionarlo con los impactos de las configuraciones espaciales de la volumetría y su vinculación con el clima local (aspectos cuantitativos) y, además, comparar mediciones de iluminación natural con las respuestas subjetivas de los usuarios en espacios abiertos (aspectos cualitativos). De esta forma, se presentan avances del estudio observacional en términos de confort visual en recintos urbanos de climas soleados, puntualmente de la ciudad de Mendoza, teniendo en cuenta la influencia de las componentes de la iluminación natural (directa, difusa y reflejada) para la estación de verano. De este modo, se pretende encontrar relaciones paramétricas que influyan en el confort lumínico del espacio urbano. 


\section{Metodología}

\subsection{Selección de casos de análisis}

Dentro de la trama de la ciudad, se identificaron dos recintos urbanos que presentan distintas permeabilidades a la luz solar. Se ubican en un Canal Vial Urbano (CVU) de alta densidad edilicia. Se trata de la Peatonal Sarmiento, situada en el microcentro
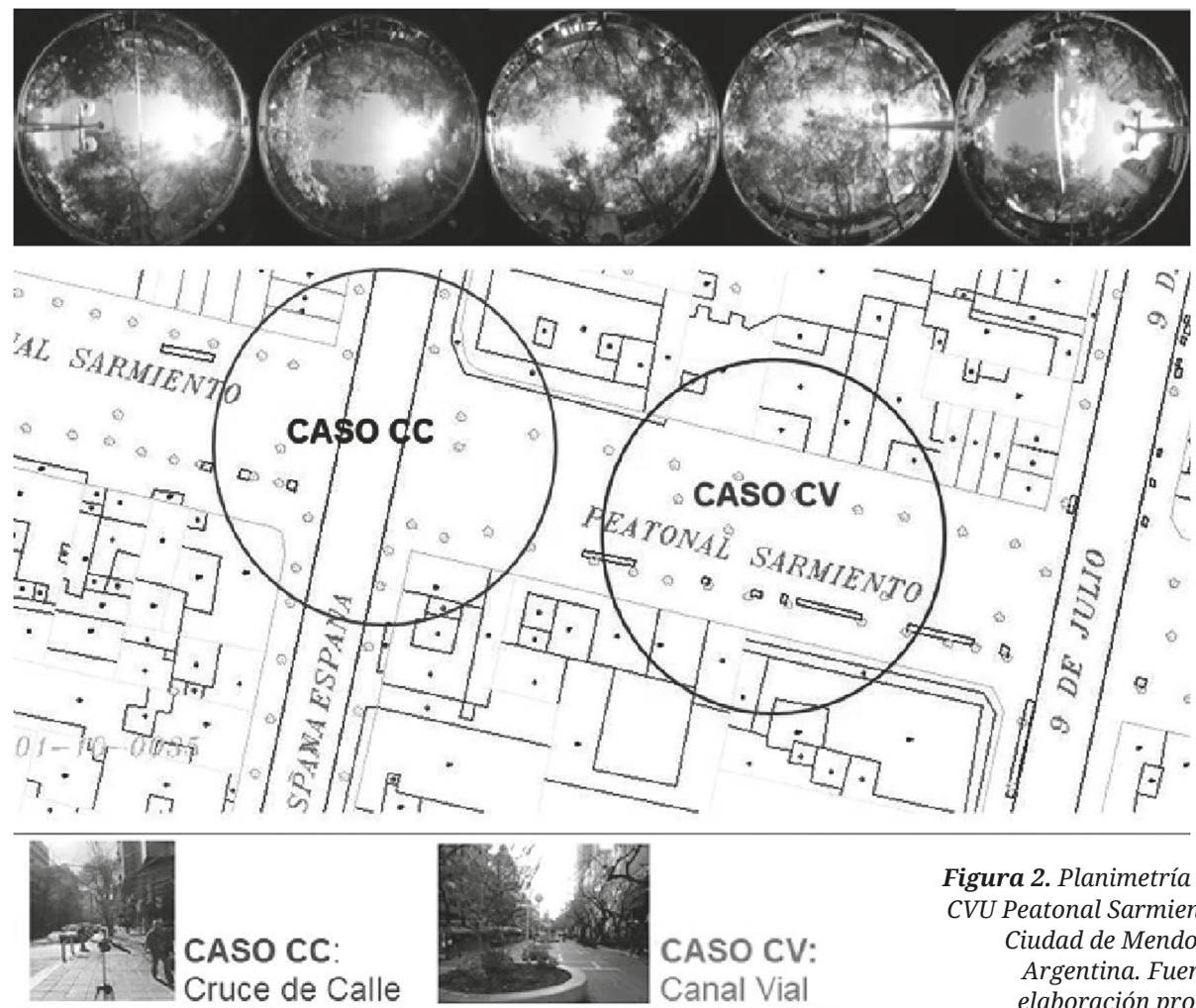

Figura 2. Planimetría del CVU Peatonal Sarmiento. Ciudad de Mendoza, Argentina. Fuente: elaboración propia 
de la ciudad de Mendoza, que se define en un eje de orientación este-oeste, de 30 $\mathrm{m}$ de ancho. Su configuración espacial de fachadas está constituida por edificios de entre tres a nueve niveles, con perfiles heterogéneos en alturas (figura 2). La especie forestal es Morus alba "morera", del tipo caducifolia, cuyos índices de transmisividad corresponden a 31,4 \% para el verano y 66,4 \% para el invierno (CAntón, Cortegoso y De Rosa, 1994: 219-230). Resultan ejemplares adultos de segunda magnitud que alcanzan alturas de 12 metros. La distancia de plantación varía entre 5,50 y 6,30 m. El estado vegetativo es bueno a regular, y los individuos forestales no alcanzan a consolidar el túnel arbóreo típico.

El primer caso, denominado caso de estudio $C V$, corresponde al sector circunscripto en el centro de la calle, que presenta un perfil urbano más cerrado, conformado por el sistema de fachadas, calzada y arboleda, además del mobiliario urbano. El segundo sector, caso de estudio $C C$, se localiza en el cruce de calles, con una configuración espacial mucho más abierta, ya que se sitúa en la intersección de calles de las manzanas, donde disminuye el grado de enmascaramiento de la edilicia y la arboleda.

\subsection{Análisis del comportamiento de luz diurna: aspectos cuantitativos}

Se realizaron mediciones de parámetros climáticos de radiación solar y luz natural, durante una jornada completa (de 9 a 18 horas) correspondiente a los días 19, 20 y 21 de diciembre, con cielo despejado para la condición invernal. Los datos climatológicos fueron monitoreados con registros cada cinco minutos, lo que permitió conformar una base de información muy significativa. En este análisis, se obtienen datos del clima local (datos globales de la región), para ser cotejados con los monitoreados en cada caso de estudio (in situ) de manera simultánea.

\subsubsection{Mediciones de parámetros lumínicos globales en exterior}

Se obtuvieron datos de Radiación Horizontal Global (IR-glo) y Radiación Horizontal Difusa (IR-dif). Como parámetros representativos de las condiciones de luz natural, se consideraron la Iluminancia Horizontal Global (EH-glo) y la Iluminancia Horizontal Difusa (EH-dif). Fueron obtenidos de la Estación de Mediciones de Iluminación Natural CCT Mendoza, incorporada a la red mundial de la CIE (Comisión Internacional d' Eclairage). Se trata de datos recolectados de manera continua en un punto base sin obstrucción (figura 3a). 
2.2.2. Medición de parámetros lumínicos in situ para los casos de estudio

Las mediciones in situ se establecieron según protocolo de mediciones (Córica y PATTINI, 2005: 05.85-05.90). Para la toma de datos de radiación solar, se utilizaron dos estaciones meteorológicas móviles marca $\mathrm{HOBO} \otimes$, modelo H21-001, equipadas cada una con un piranómetro de silicona S-LIB-M003, localizados a 1,50 $\mathrm{m}$ de altura (figura $3 \mathrm{~b}$ ). El rango operativo se encuentra entre $-20 \mathrm{y}+50^{\circ} \mathrm{C}$. Las iluminancias fueron relevadas con fotómetros LI-210SA Licor 21, sistema combinado UTA / HOBO U12-013 como datalogger.

\subsection{Respuestas de los usuarios a las condicionantes lumínicas de los espacios: aspec- tos cualitativos}

Para relacionar las sensaciones de los usuarios con las características físicas del campo luminoso, se hicieron preguntas específicas a las personas en los espacios abiertos. Como parámetros representativos para su evaluación lumínica, se registraron mapeos de luminancias que contienen información de los ambientes visuales en términos de reflectividad incidente en el campo visual, además del registro de luz proveniente del entorno construido, en este caso la iluminancia cilíndrica.

Las evaluaciones subjetivas se procesaron estadísticamente mediante la técnica de Diferencial Semántico. El método es una escala de clasificación que mide el significado subjetivo que los estímulos provocan en los sujetos. Contiene dos elementos fundamentales: conceptos y escalas bipolares.

Las preguntas fueron incluidas en un cuestionario donde se consultaron aspectos relacionados con el confort visual, tales como preferencia de cantidad de sol; nivel de luz en el espacio; sensibilidad al deslumbramiento: nivel de tolerancia y superficie de procedencia de los brillos o reflejos molestos, entre otras. Las encuestas se efectuaron de manera personalizada a lo largo de la jornada, y se tuvo en cuenta el horario de las entrevistas para el entrecruzamiento de las variables y la correlación con las mediciones objetivas. En el caso de estudio CC se obtuvo una muestra de $130 \mathrm{~N}$ (personas encuestadas), mientras que en CV el tamaño de la muestra fue de $167 \mathrm{~N}$.

Los mapeos de luminancias se confeccionaron a partir de fotografías de Amplio Rango Dinámico (HDRI) (INANici \& GALVIN, 2004). Las imágenes se tomaron cada una hora con cámara Nikon Coolpix 5400 y lente Nikon FC-E9, y fueron procesadas por medio del software 


\section{Luz solar en recintos urbanos de alta densidad edilicia en el modelo oasis \\ de la ciudad de Mendoza. Aspectos cuantitativos y cualitativos (período estival)}

Photosphere-MAC OS. Este software combina todas las imágenes de bajo rango dinámico (LDR) en una de alto rango, correspondiendo cada pixel a un valor fotométrico de luminancia. La calibración de las imágenes se obtiene con luminancias de control a través de Luminancímetro Minolta LS 110.

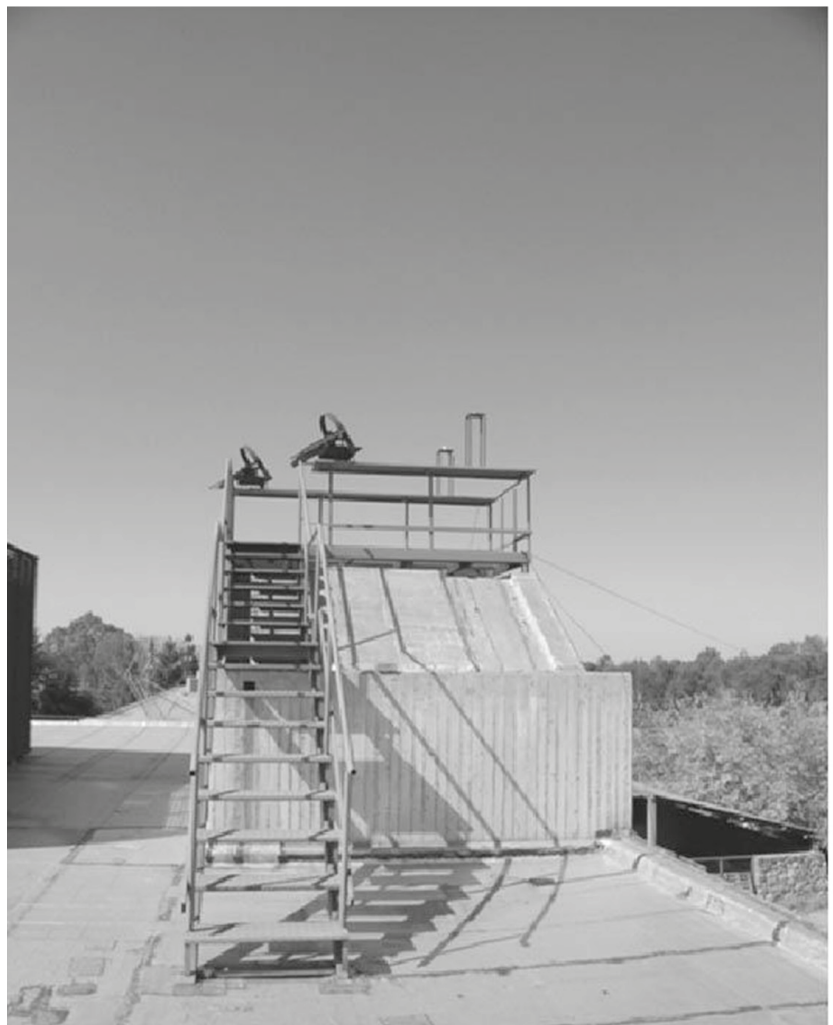

(a)
Figura 3.

(a) Estación de mediciones CCT-Mendoza.

(b) Disposición de equipamiento in situ. (c) Cabezal y sensor de Iluminancia cilíndrica. Fuente: elaboración propia (b)

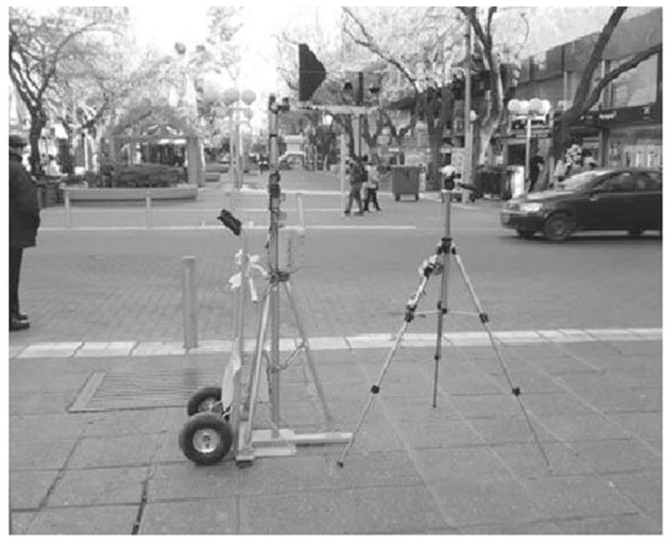

(c)

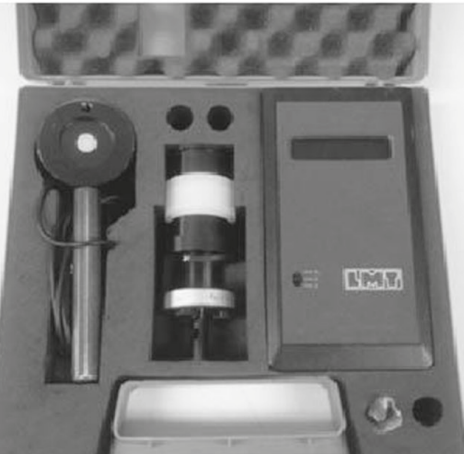


La iluminancia horizontal no considera la luz reflejada del entorno construido, en especial las provenientes de las fachadas que afectan una gran parte del campo visual de los usuarios (Compagnon, 2000). La magnitud que incide en los ojos de las personas como principales receptores verticales está dada por la Iluminancia cilíndrica (Ecyl), que incluye la cantidad de luz vertical proveniente de todas las direcciones. Por lo tanto, está relacionada con las sensaciones de los usuarios con respecto a las características del entono lumínico. Para ello, se utilizó un luxímetro marca LMT con cabezal para medición de iluminancia cilíndrica, ubicado a la altura del observador de 1,50 m (figura 3c).

\section{Análisis de resultados}

\subsection{Aspectos cuantitativos}

Como se observa en las imágenes hemisféricas de la figura 4, las condiciones lumínicas de ambos recintos se encuentran condicionadas no solo por las variables geométricas y morfológicas del ambiente construido, sino y fundamentalmente por la morfología del verde urbano en su máxima expresión foliar.
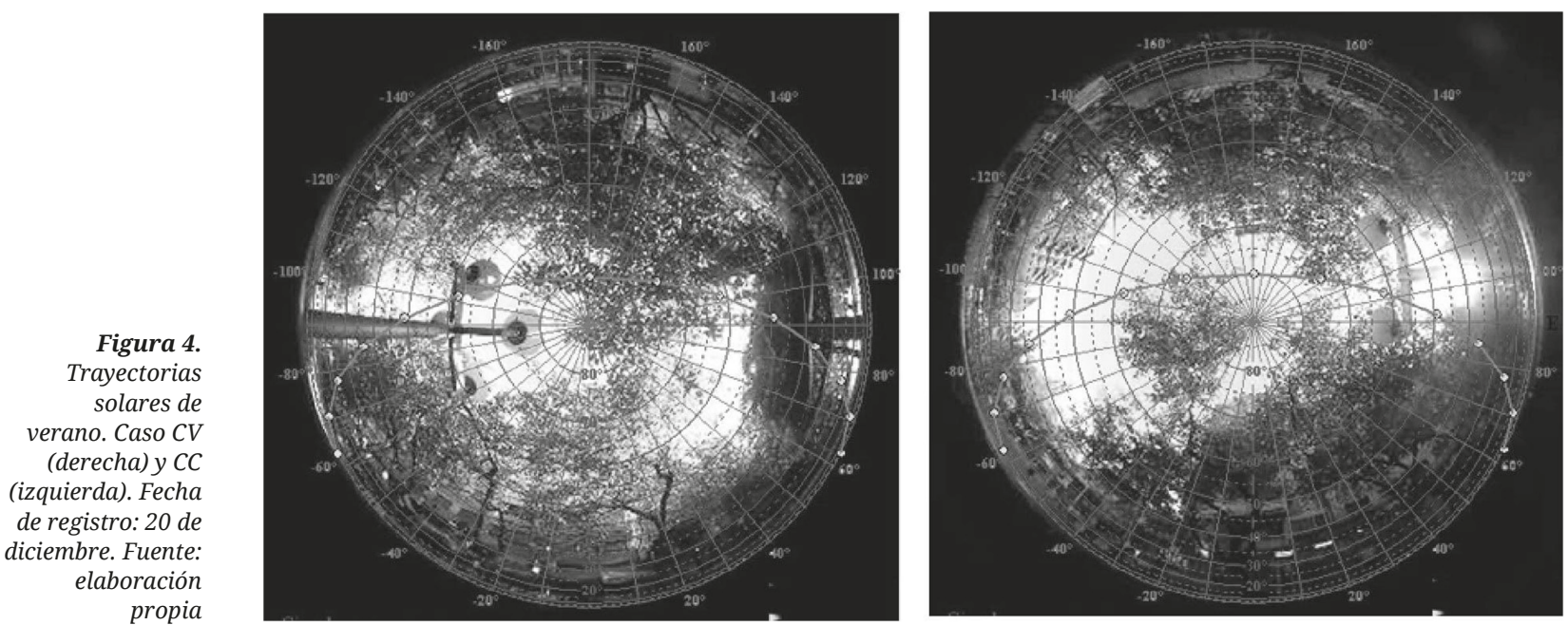


\section{Luz solar en recintos urbanos de alta densidad edilicia en el modelo oasis \\ de la ciudad de Mendoza. Aspectos cuantitativos y cualitativos (período estival)}

La figura 5 coteja los valores de radiación solar de la región y los registrados en los escenarios urbanos, y muestra los mayores rangos de global para el año $\left(1100 \mathrm{~W} / \mathrm{m}^{2}\right.$ aproximadamente). Estos efectos están dados principalmente por los valores de los ángulos solares en el solsticio de verano, situación que impacta con mayor intensidad en la región. Sin embargo, en el Caso CV la radiación solar se ve condicionada por períodos. Es el caso de niveles mínimos en horas de la mañana (de 9 a 11), bloqueados por el follaje y la edilicia. Hasta las 15 horas se muestra una exposición directa del sol $\left(1000 \mathrm{~W} / \mathrm{m}^{2}\right)$, lo que resulta de la ubicación del sensor en una zona de vano de follaje, ante la falta de continuidad de túnel verde. Desde las 16 en adelante, los rangos decaen a partir de las obstrucciones que ejercen la volumetría circundante y la permeabilidad de la especie forestal. El Caso CC presenta el mayor aporte de radiación solar entre las 12:30 y las 18 horas, correspondiente a la apertura de calle, y llega a niveles similares a los registrados en la estación meteorológica. Esta diferencia en duración (seis horas y media en el Caso CC versus cuatro horas en el Caso CV) se debe fundamentalmente a las diferencias urbano-arquitectónicas de los casos (centro de la cuadra- intersección de calles).
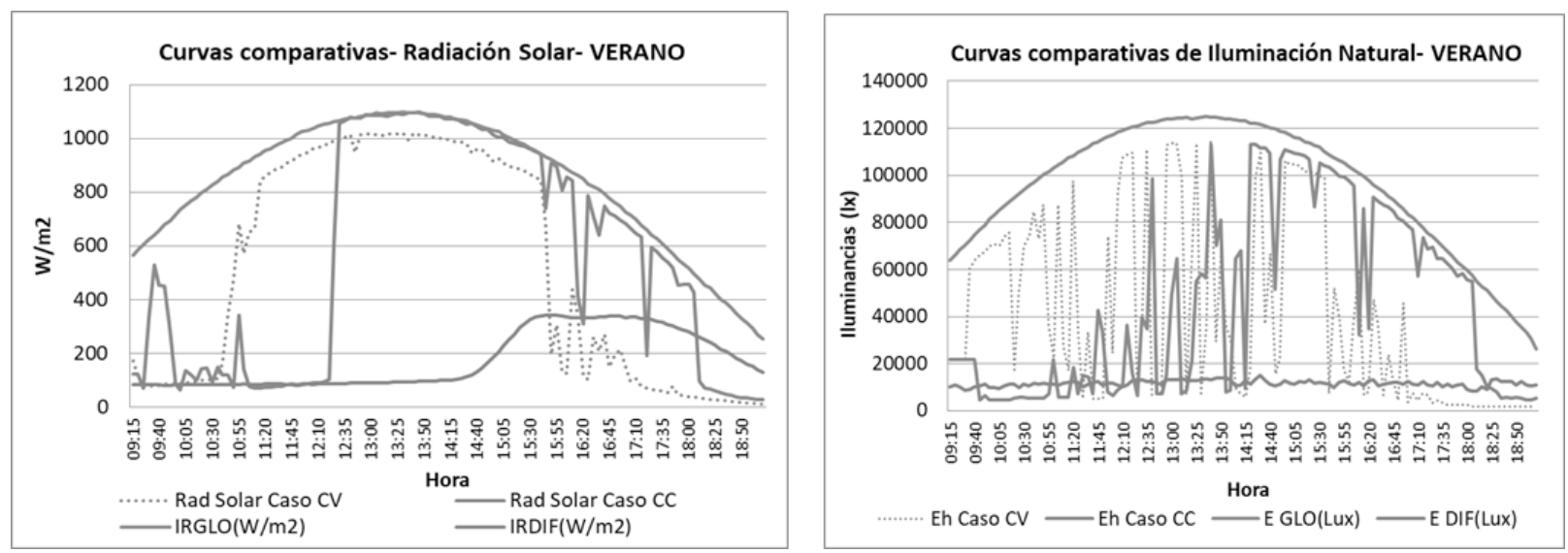

Figura 5. Valores de radiación solar comparativos para los casos de estudio. Fecha de registro: 20 de diciembre. Fuente: elaboración propia 
Desde el análisis de Iluminación Natural (figura 6) también se corroboran para el verano las mayores intensidades luminosas anuales. Como valores globales máximos obtenidos en la estación se exponen: 125000lx de EH-glo registrados al mediodía solar y 15000lx aproximadamente de EH-dif, con un comportamiento constante a lo largo de la jornada (media: 11600lx). En ambos casos, se producen cambios drásticos y oscilantes en las curvas. La presencia de la arboleda en su máxima expresión foliar define para CV períodos en sol y en sombra proyectados por la trama del follaje. Se detectan picos muy altos a partir del ingreso de luz directa entre huecos o vanos de los árboles (Eh máx: 114400 lx) y valores heterogéneos de luz difusa, filtrada por los diferentes grados de permeabilidades del follaje. Estos rangos oscilan entre un valor mínimo de 7000 lx y un máximo de 40000 lx, según el recorrido de la dinámica solar diurna.

En el caso de análisis CC, la mayor parte de la mañana presenta condición de sombra. Las iluminancias se muestran muy bajas (5000 lx), y en este caso cabe puntualizar que esta situación se produce por un doble efecto de sombreamiento: no solo por la obstrucción de la volumetría de la fachada norte del canal vial, sino también por la obstrucción que ejerce la copa de los árboles. A partir del mediodía se van registrando incrementos progresivos a medida que los ángulos solares van aumentando. Las iluminancias alcanzan valores máximos de luz directa de 113000 lx al mediodía solar, los cuales van decreciendo en horas de la tarde, presentando situaciones de sol y de sombras por las diferencias de alturas de la volumetría edilicia en ambas fachadas.

Las distribuciones de iluminación natural obtenidas para la condición de verano destacan claramente la figura del arbolado como principal elemento de obstrucción y control, ante la incidencia de los rayos solares sobre la superficie horizontal. Esto se puede corroborar a partir de los estudios que se realizaron durante el invierno en los mismos casos.

\subsection{Aspectos cualitativos}

\subsubsection{Mapeos de luminancias}

Desde el punto de vista del ambiente visual, las reflectancias de las superficies y sus variaciones afectan el confort visual de los usuarios, y en este punto se deben contemplar aspectos o dimensiones temporales, espaciales y de intensidades lumínicas de la luz solar (BRotAs y WIENOLD, 2014: 688-693). 
En tareas al aire libre, los brillos o reflejos que se registran en el sistema visual son de gran importancia para evaluar la magnitud de deslumbramientos, lo que genera deterioro de la visión ${ }^{3}$. En espacios exteriores y en muchas ocasiones el deslumbramiento está dado por la luz solar reflejada (superficies metálicas o revestimientos claros), cuyos efectos son impulsados por las propiedades de la superficie o incluso provenientes desde la fuente directa como el sol (BROTAS y WiLSON, 2002: 703-708).

En las figuras 6 y 7, para cada fotografía de ojo de pez, se indican los valores de luminancia. Estos valores son resultantes de las inter-reflexiones de los materiales y de la incidencia de iluminación natural debidas a los rayos de sol (luz directa) y a la luz difusa proveniente del cielo, de la tierra y de los edificios circundantes. La exposición al sol determina que en verano las luminancias se presenten más relacionadas, al estar controladas por la masa de verde, siendo el elemento responsable de condicionar las intensidades luminosas emitidas por las superficies iluminadas. Puede observarse que las reflectancias preponderantes se corresponden con la fuente solar ubicada en las zonas sin conformación de túnel o vanos en el follaje y de los pavimentos del suelo, pero dada la temporalidad del recurso, no se mantienen constantes a lo largo del día, por lo que los deslumbramientos pueden darse de manera ocasional en las personas.

Los mapeos obtenidos para las principales escenas del día exhiben importantes contrastes y cambios lumínicos en el caso CV (figura 7). Uno de los principales factores que determina estas condiciones está definido por la estacionalidad de las especies forestales, lo que evidencia la discrepancia en las superficies de asoleamiento y de sombra en cada escenario por la temporalidad solar y el ingreso de rayos entre los vanos propios del follaje (Reflectancia mín.: $160 \mathrm{~cd} / \mathrm{m}^{2}$ y máx: $7400 \mathrm{~cd} / \mathrm{m}^{2}$.)

En el caso del CC (figura 7), las reflectancias de las superficies determinan un contexto visual contrario a CV, más allá de que ambos espacios urbanos corresponden al mismo cañón vial. Si se tiene en consideración la dimensión temporal, a lo largo de la jornada, las personas están expuestas a elevadas luminancias provenientes de la fuente directa del sol, pero a la vez el espacio presenta una conformación morfológica abierta definida por el cruce de calle. La contribución de luminancias del suelo resulta de gran importancia (superiores a los $5000 \mathrm{~cd} / \mathrm{m}^{2}$ ), con un alto poder reflectante. Dadas las características edilicias, los muros pierden fuerza como superficies reflectantes de brillos. Debido a las
3. Según la CIE (Comission Internationale de Léclairage), se define como deslumbramiento a la condición de la visión en la que hay incomodidad o una reducción en la capacidad de ver detalles $u$ objetos, causados por una distribución inadecuada o el rango de luminancia o por contrastes extremos. En tareas al aire libre, los brillos o reflejos que se registran en el sistema visual son de gran importancia para evaluar la magnitud de deslumbramiento discapacitante o perturbador, como el deterioro de la visión, situación que puede causar accidentes. El deslumbramiento discapacitante es un efecto causado por la dispersión de la luz en el ojo, que reduce el contraste de luminancias de la imagen en la retina. 

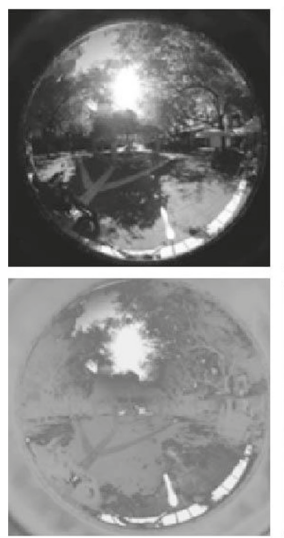

$10 \mathrm{hs}$
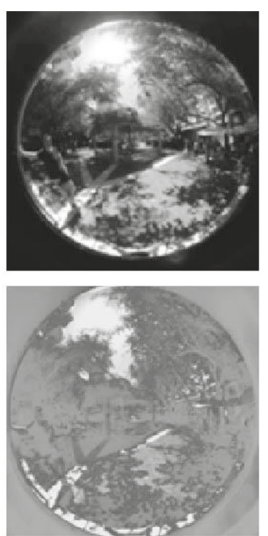

12hs
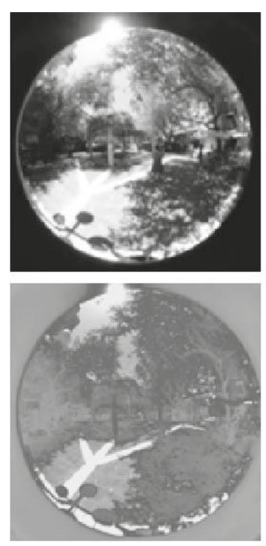

14hs
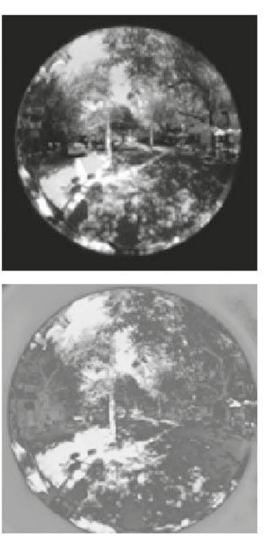

16hs
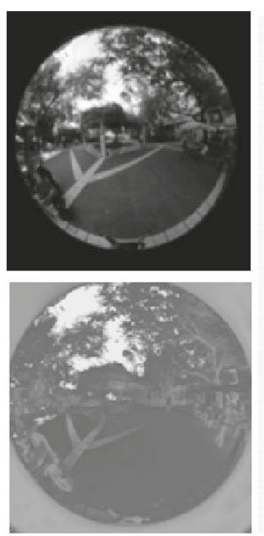

18hs

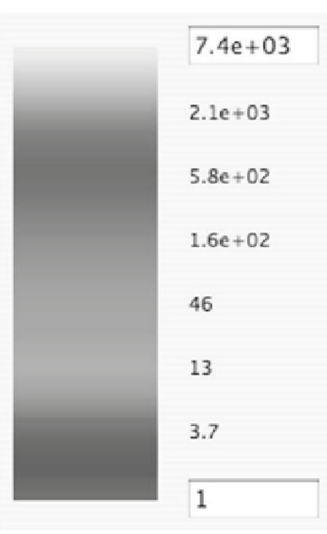

Figura 6. Imágenes HDR y mapeos de luminancias ( $\mathrm{cd}$ ) m2)- Caso CV. Fecha de registro: 20 de diciembre. Fuente: elaboración propia altas intensidades, provenientes de ambas fuentes, se puede suponer que la reflexión solar dentro del campo de visión generará deslumbramientos. Por lo tanto, se estima que en espacios urbanos de estas características, en el período de verano y según los ángulos de incidencia solar cercanos al mediodía, pueden ocurrir deslumbramientos en las personas.

\subsubsection{Niveles de iluminancia cilíndrica}

Las curvas de Ecyl obtenidas en la figura 8 confirman los escenarios visualizados en ambos casos. Estos corroboran de manera clara que la condición del centro de CVU presenta mayores variaciones lumínicas producto de las permeabilidades de la arboleda. Mientras que, para el entorno de cruce de calle, la máxima registrada supera los 20000lx, dándose un incremento con una mayor variabilidad en los rangos de iluminancia horizontal, con un comportamiento proporcional en la cilíndrica (tabla 1). Esto se produce como consecuencia de la temporalidad de la luz solar y el ingreso de la componente directa y la proyección de sombras por los perfiles construidos. 

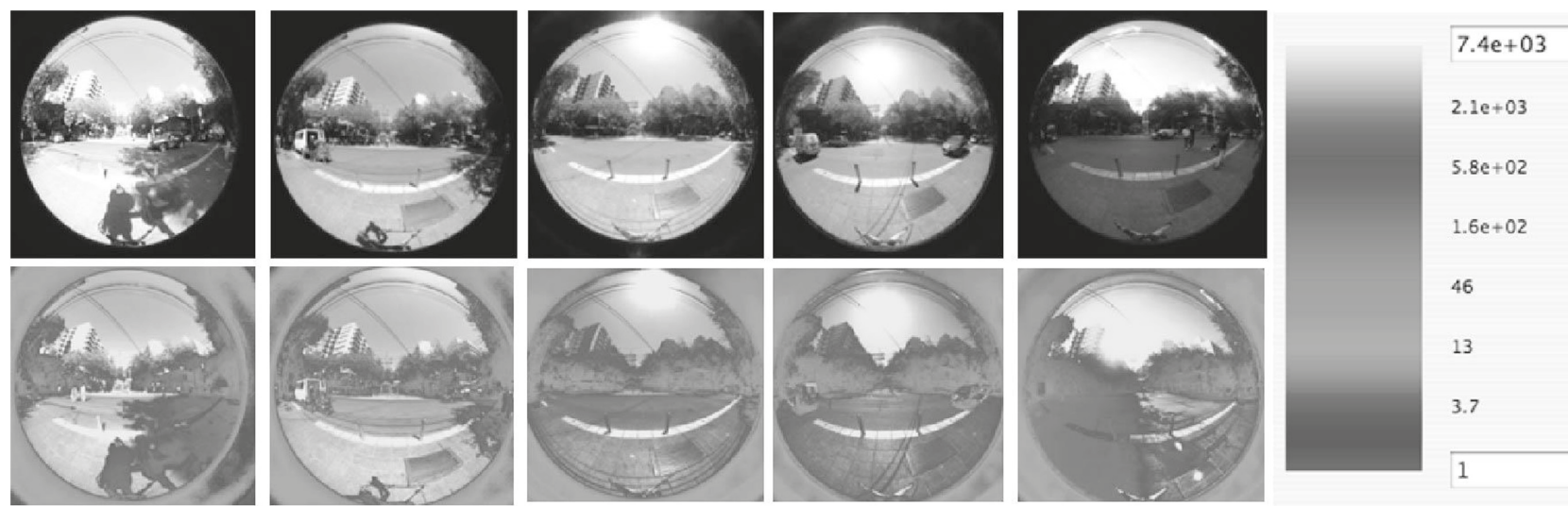

$2.1 e+03$

$5.8 \mathrm{e}+02$

1. $6 \mathrm{e}+02$

46

13

3.7

1

$10 \mathrm{hs}$

$12 \mathrm{hs}$

14hs

16hs

18hs

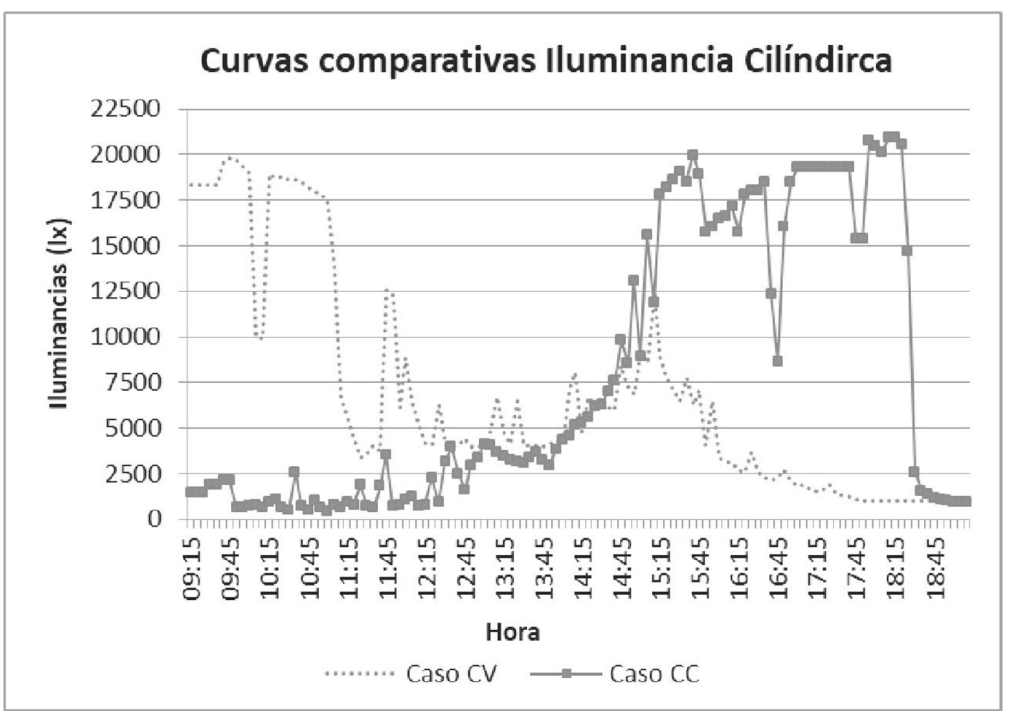

Figura 8. Registro comparativo de iluminancias. Fecha de registro: 20 de diciembre. Fuente:

elaboración propia

Figura 7. Imágenes HDR y mapeos de luminancias ( $\mathrm{cd} /$ m2). Caso CC. Fecha de registro: 20 de diciembre. Fuente: elaboración propia 
Tabla 1

Estadísticos descriptivos de iluminancia cilíndrica para cada caso de estudio (lx).

\begin{tabular}{l|c|c|c|c|c} 
& N & E Mínimo & E Máximo & E Media & Desviación típica \\
\hline Caso CC & 130 & 440 & 20750 & 10595 & 7437 \\
\hline Caso CV & 167 & 300 & 19780 & 10040 & 5652
\end{tabular}

Fuente: elaboración propia

\subsubsection{Sensación de cantidad de sol}

Inicialmente y como parte de la información que testear, se consultó a los usuarios su opinión sobre la cantidad de sol disponible en el espacio. Las respuestas para el caso CV arrojaron que el $51 \%$ preferiría menos sol y el $46 \%$, que está bien. Los ocupantes no expresan variaciones de las percepciones, debido a que el espacio se encuentra en condiciones lumínicas homogéneas dadas por la obstrucción que la volumetría y la arboleda ejercen en el entorno.

En cuanto a las personas testeadas en el cruce de calle (CC), el $50 \%$ preferiría menos sol, mientras que para el $48 \%$ está bien, datos sorpresivos teniendo en cuenta que este caso representa mayores intensidades lumínicas dadas la amplitud y visión de cielo del sector. Esto podría indicar adaptación al medio de los habitantes locales.

\subsubsection{Percepción de la luz natural}

Los resultados preliminares obtenidos sobre la sensación luminosa de los espacios exteriores bajo la incidencia de diferentes condiciones de la luz natural han determinado que las opiniones de los encuestados aparezcan como similares, a pesar del hecho de que los contextos lumínicos sean diversos e incluso adversos en algunos casos. En ninguno de los dos casos de estudio, las personas reportan percibir una cantidad de luz natural insuficiente. La mayoría (el 60 \%) coincide en que ambos casos tienen "suficiente" luz natural. En el caso CC (figura 9a), el $11 \%$ de las personas advierte una iluminación "excesiva”, mientras que en el caso CV (figura 9b), ese porcentaje es solo del $6 \%$.

En términos generales, las valoraciones subjetivas de la mayoría de los usuarios han calificado a la cantidad de luz en el campo luminoso como "suficiente". Estos resultados en cierta 

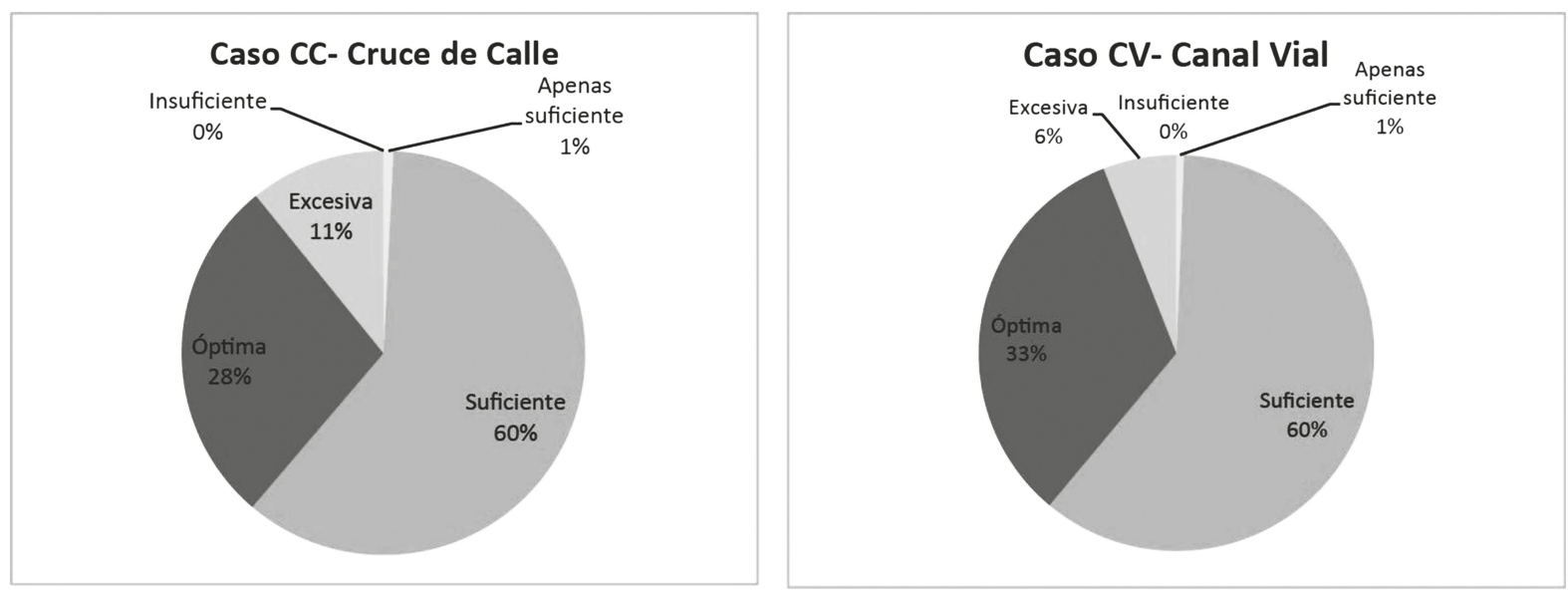

Figura 9. Voto de sensación de luz natural. Verano. Fuente: elaboración propia

forma establecen que es relativo definir cualitativamente el nivel de iluminación mediante estas técnicas de observación, a partir de la percepción que las personas expresan ante las consignas consultadas. Por ejemplo, en el caso del centro de calle, en términos de eficiencia visual, a la hora de realizar tareas visuales como desplazarse o la simple permanencia en el espacio, las condiciones lumínicas aparecen con los rangos más bajos de iluminancias registrados, y sin embargo, representan niveles "suficientes y óptimos" (asociados a mucha luz) en términos de preferencias. Mientras que, en el espacio más abierto, resultan las mayores intensidades lumínicas a lo largo del día y las preferencias de las personas coinciden con niveles suficientes, e incluso en casos de disconfort visual como deslumbramiento.

\subsubsection{Percepción de reflejos y fuentes de deslumbramiento}

Finalmente, en cuanto al procesamiento estadístico propuesto para la sensibilidad de las personas a deslumbramientos en la correlación correspondiente al caso CV, casi la totalidad de la muestra ( $81 \%$ ) no percibe molestias por deslumbramientos a partir de brillos o reflejos (figura 10a), respuesta razonable y consecuente con los rangos lumínicos que presenta el entorno urbano. En cuanto a la fuente de deslumbramiento (figura 10b), en el rango de menor Ecyl menor, solo se reportan deslumbramientos provenientes del sol y del 
piso. En el rango intermedio, aparece el equipamiento urbano y en el rango de mayor Ecyl también se menciona la edilicia, puntualmente de las superficies vidriadas en la fachada con exposición al norte.

En este punto, cabe aclarar que el $93 \%$ de las personas encuestadas no presentaba ningún tipo de protección solar, como lentes de sol o gorras, y que dada la presencia de sombras tampoco se manifestaron gestos o movimientos para apantallar sus ojos del exceso de luz (por ejemplo, disposición de las manos sobre los ojos, girar o doblar la cabeza, parpadeos).

En el espacio CC, el 83 \% no percibe sensación de deslumbramiento (figura 11). En este punto sorprende ver que los sujetos se encuentran bajo la incidencia de luz directa en el campo visual (20000lx). Esto denota o confirma probablemente el efecto de adaptación de las personas al clima luminoso regional. El porcentaje de personas a las que les molesta el deslumbramiento es levemente superior (10 \%) al caso CV. En el rango de Ecyl inferior (menos de 1900 lx), ninguna persona manifestó percibir deslumbramiento. En el rango medio, se perciben deslumbramientos provenientes del sol (10\%), equipamiento urbano,
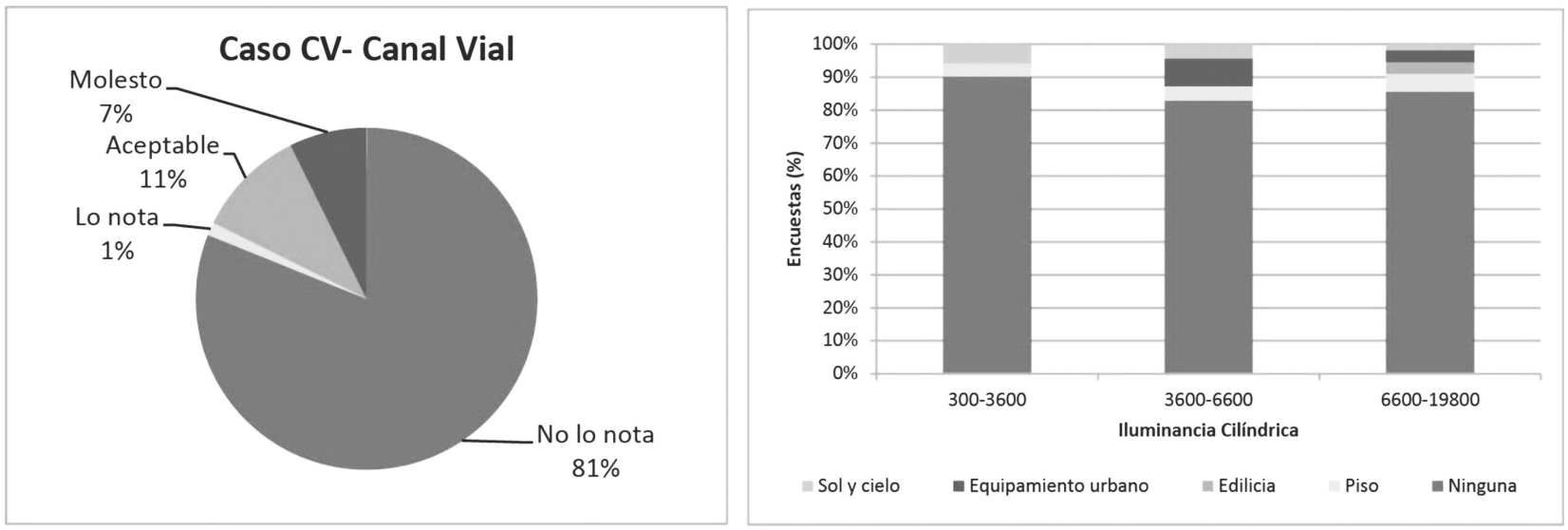

Figura 10. Porcentajes de deslumbramiento (a) y de reflejos provenientes del entorno (b). CV. Fuente: elaboración propia 

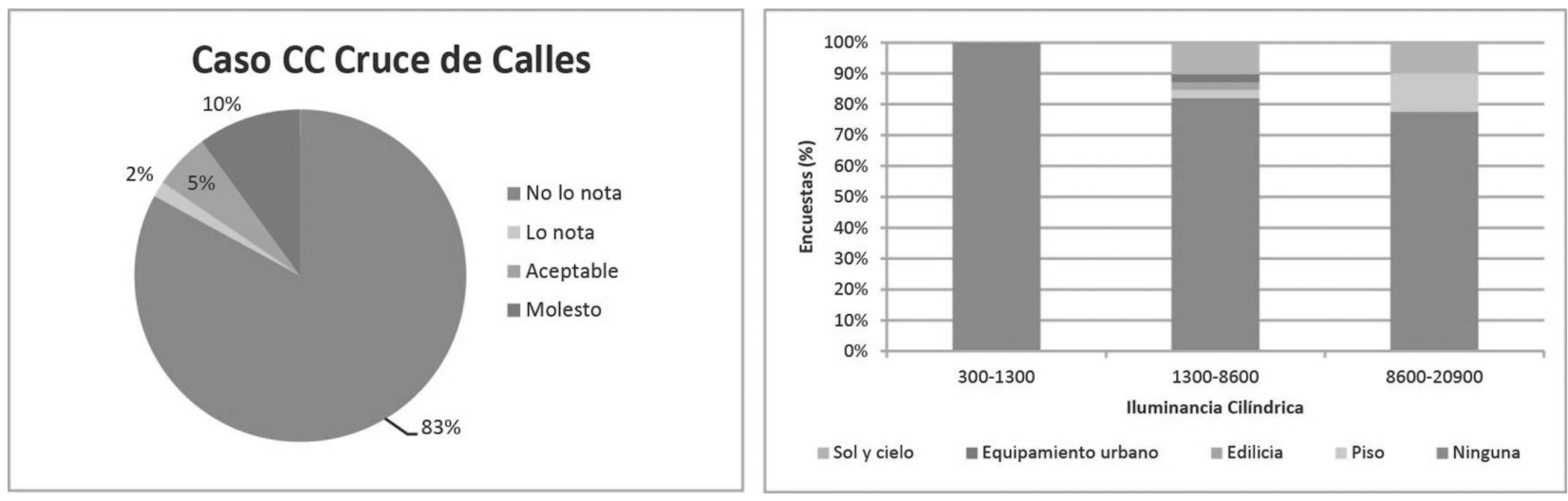

Figura 11. Porcentajes de deslumbramiento (a) y de reflejos provenientes del entorno (b). CC. Fuente: elaboración propia

edilicia y piso, mientras que en el rango mayor, el sol y el piso representan las principales fuentes de deslumbramiento, $13 \%$ y $10 \%$, respectivamente.

Si bien hay un aumento importante en las reflectancias provenientes del piso (figura 7), para las personas encuestadas no representa una fuente de deslumbramiento.

\section{Conclusiones}

La ciudad de Mendoza presenta una estructura urbana de modelo oasis dentro de un contexto desértico, donde - a diferencia de las formas tradicionales de regiones áridas- el efecto de la intensa radiación es controlado estacionalmente con la presencia de la arboleda urbana y de la morfología edilicia. En este trabajo se presentaron resultados de la evaluación integral de la luz solar y del confort visual en entornos urbanos típicos de la alta densidad edilicia correspondientes a este modelo urbano, para la estación de verano, como parte de un estudio anual. Este además permitió analizar de qué manera se correlacionan parámetros subjetivos de las personas, en términos de preferencias, con aspectos físicos o cuantitativos según las diferentes componentes de la iluminación natural. 
Desde el punto de vista lumínico, el análisis cuantitativo arrojó que los comportamientos de la luz natural responden claramente a las configuraciones espaciales. Se determinó que en recintos urbanos arbolados de alta densidad edilicia en zonas áridas, existe un predomino de luz difusa generada principalmente por la proyección de sombras provenientes de la canopia vegetal, mientras que en configuraciones más abiertas, como cruces de calles, las condiciones son variables por contrastes de luz directa y difusa (obstrucciones de la edilicia) según las trayectorias solares diarias. Se comprueba una vez más que la radiación solar resulta determinante del comportamiento lumínico de los ambientes urbanos. Se valora especialmente la combinación entre volumetría y forestación como factor clave a la hora de mitigar o disminuir esta radiación incidente en la estación calurosa.

En cuanto a los aspectos cualitativos, los mapeos de luminancias muestran que las distribuciones lumínicas superficiales obtenidas no son homogéneas. Esto plantea la necesidad de medir y evaluar la distribución de luminancias no uniformes y sus cambios en el espacio y en el tiempo. El aumento de la obstrucción de la edilicia afecta principalmente a la luminancia de los espacios urbanos.

Las opiniones resultantes de las valoraciones subjetivas, en la mayoría de las encuestas, han determinado que la cantidad de luz incidente en el campo visual de las personas expone niveles suficientes de luz ya sea para desplazarse o permanecer en los espacios exteriores, más allá de las diferencias lumínicas que se puedan generar en el escenario urbano.

Respecto de la presencia de sol, puede interpretarse que más allá de la diversidad de escenarios de luz (directa-difusa), los sujetos se encuentran adaptados o habituados al clima luminoso de la región y, consecuentemente, a la multiplicidad de contrastes que se generan en el espacio urbano.

Se propone a futuro seguir trabajando en la interpolación de aspectos lumínicos con térmicos, y de esta forma poder confeccionar una cartilla de recomendaciones y pautas como propuestas de diseño urbano. Resulta imprescindible también poder realizar estudios específicos con experimentos psicofísicos en cuanto a rendimiento, eficiencia y eficacia en el uso de los espacios abiertos. 
Bibliografía

APPLE, Marianne, APPLE, Loyal y BLASCH, Donald (1980) Low Vision. En Foundations of orientation and mobility. R. L. Welsh y B. B. Blasch (Eds.). New York.

BOYCE, Peter y SANFORD, Linda (2000). "Lighting to enhance visual capabilities". En The Lighthouse Handbook on Vision Impairment and Vision Rehabilitation. Rosenthal y E. E. Faye (Eds.). York: Oxford University Press.

BROTAS, Luisa y WILSON, Wout (2002). "Reflected sunlight in urban canyons. Towards a new approach". Ponencia presentada en EPIC 2002 AIVC Energy Efficient \& Healthy Buildings in Sustainable Cities - The 3rd European Conference on Energy Performance \& Indoor Climate in Buildings. Lyon, France.

BROTAS, Luisa y WIENOLD, Jan (2014). "Solar reflected glare affecting visual performance". Ponencia presentada en Windsor Conference: Counting the Cost of Comfort in a Changing World. Volume: In proceedings. Cumberland Lodge, Windsor Park, UK, London.

CANTALEJO CANO, José (2001). "Prescripción de filtros: procedimientos de observación sistemática y procesode toma de decisiones”. En Integración: Revista sobre ceguera y deficiencia visual. N. ${ }^{\circ} 37$.

CANTÓN, M. Alicia, CORTEGOSO, J. Luis y DE ROSA, Carlos (1994). "Solar permeability of urban trees in cities of western Argentina”. En Energy and Buildings, N. ${ }^{\circ} 20$.

CIE COLLECTION ON GLARE (2002). "Equation for disabilityglare" 146 CIE C 1-50 report and Glare from small, large and complex sources, 147 CIE TC 3-01 report Comission Internationale de Léclairage.

COMPAGNON, Raphael (2000). "Solar and Daylight availability in urban areas". PRECis project Final Technical Report, Ecole d'ingénieurs et d'architectes de Fribourg.

COMPAGNON, Raphael (2004). "Solar and daylight availability in the urban fabric".En:Energy and Buildings, N. ${ }^{\circ} 36$.

CóRICA, Lorena (2010). "Comportamiento de la luz natural en entornos urbanos representativos del modelo oasis en regiones áridas. Caso de estudio: ciudad de Mendoza”. Tesis doctoral. Departamento de Luminotecnia, Luz y Visión Herberto Büller. Tucumán, Universidad Nacional de Tucumán.

CóRICA, Lorena y PATTINI Andrea (2005). "Protocolo de mediciones de iluminación natural en recintos urbanos”. En Avances en Energías Renovables y Medio Ambiente. Volumen 3, N. ${ }^{\circ}$. 
FONTOYNONT, Marc, TSANGRASSOULIS, A. \& SYNNEFA, A. (2004) Daylighting. In: SynthLight Handbook. Eurepean Comision, Bruselas.

GOYETTE, Joëlle y COMPAGNON, Raphaël (2004). "R. RUROS- Rediscovering the Urban Realm and Open Spaces Project: two case studies in Fribourg, Switzerland". Ponencia presentada en ICUC-5 Fifth International Conference on Urban Climate, Lodz, Poland.

GUZOWSKI, Mary (2001) Daylighting for sustainable design. McGraw Hill, New York.

INANICI, M., GALVIN J. (2004). "Evaluation of High Dynamic Range photography as a luminance mapping technique”. LBNL Report No. 57545, Berkeley: Lawrence Berkeley National Laboratory.

MASCARÓ, Lucía (1983) Luz, clima e arquitetura. Livraria Nobel SA, SP, Brasil.

PEARLMUTTER, David; BITAN, Ariel.; BERLINER, Pedro.(1999) "Microclimatic Analysis Of ‘Compact Urban Canyons In An Arid Zone”. Atmospheric Environment 33.

PONTE, Ricardo (1987) Mendoza, aquella ciudad de barro. Municipalidad de la Ciudad de Mendoza. Mendoza.

ROMBAUTS, Patrick; VANDEWYNGAERDE, Hans y MAGGETTO, Gaston (1989). "Minimum Semicylindrical Illuminance and Modelling in Residential Area Lighting”. Lighting Research and Technology, v. 21, n. 2, p. 49-55, jan. 1989.

TREGENZA, Peter (1995). "Mean daylight illuminance in rooms facing sunlit streets”. En Building and Environment, 30 (1).

TSANGRASSOULIS, Aris; SANTAMOURIS, Mattheos; GEROS, Vasilis; WILSON, Michael y ASIMAKOPOULOS, Dimosthenis (1999). "A method to investigate the potential of south-oriented vertical surfaces for reflecting daylight onto oppositely facing vertical surfaces under sunny conditions”. En Solar Energy, ‥ ${ }^{\circ} 66$. 